\title{
A Study on Knowledge, Attitude and Practices of Obtaining Informed Consent for Medical and Surgical Procedures Among Medical Officers in Teaching Hospital Kalubowila and Karapitiya, Sri Lanka
}

Lelwala PBW*, Dasanayaka PB

Office of the Judicial Medical Officer, Colombo south teaching hospital, Kalubowila, Sri Lanka

\begin{abstract}
Introduction: At present most of the patients are educated, knowledgeable and actively participating in medical decision making. However, the literature on medical officers' knowledge, attitudes, and practices, as well as informed consent, in Sri Lanka is quite limited and our study is carried out to fulfil this gap.
\end{abstract}

Methodology: A cross-sectional descriptive study was conducted among randomly selected 404 medical officers at two Teaching Hospitals in Sri Lanka.

Results: Among the study group of total 404 medical officers, $57 \%(\mathrm{n}=232)$ were males and $82 \%(\mathrm{n}=331)$ were Grade Medical Officers. Ninety five percent $(n=384)$ of them knew that informed consent should be obtained in vaginal examination and rectal examination and 98\% $(\mathrm{n}=396)$ of them knew and apply the knowledge into practice in the case of children and other persons who are incapable of giving consent and 97\% ( $n=392)$ of them knew that they have to consider the mental state of the patient before requesting consent. Almost all medical officers $96 \%$ $(n=348)$ believe that the doctor who carries out the treatment or medical procedures is the best person to obtain informed consent from the patient. The majority of the medical officers $70 \%(\mathrm{n}=283)$ only explain common risks. In comparison, 29\% $(n=117)$ explain all risks and only $01 \%(n=04)$ explain all risks including possible remote risks as well.

Conclusion: A reasonable number of doctors have adequate knowledge regarding informed consent but are very poor in some areas. It is highly recommended continuous professional development program and knowledge updating program covering medical ethics at each hospital level.

Keywords: Autonomy, Informed consent, , medical ethics.

Received: 15 Oct 2021, Revised version accepted: 25 Dec 2021, Published: 31 Dec 2021. *Corresponding author: Lelwala PBW, $\$ email: buddhika.lelwala@gmail.com (D) ORCID: https://orcid.org/0000-0002-6303-4289

Cite this article as: Lelwala PBW, Dasanayaka PB. A Study on Knowledge, Attitude and Practices of Obtaining Informed Consent for Medical and Surgical Procedures Among Medical Officers in Teaching Hospital Kalubowila and Karapitiya, Sri Lanka. Medico-Legal journal of Sri Lanka. 2021;9(2):14-19. DOI: http://doi.org/10.4038/10.4038/mljsl.v9i2.7445

Copyright: @ 2019 with the Medico-legal Journal of Sri Lanka.

This is an open-access article distributed under the terms of the Creative Commons Attribution 4.0 International License, which permits unrestricted use, distribution, and reproduction in any medium provided the original author and source are credited.

\section{Introduction}

As a basic component of the doctor's role, informed consent is a professional ethical concern and a legal obligation of the doctor toward the patient.[1] It is the patient's voluntary authorization of a medical action, with knowledge of the potential repercussions. A physician must advise a patient about all of the potential advantages, risks, and alternatives involved in any surgical or medical procedure so that the patient can make an informed and voluntary decision about whether or not to receive medical treatment.[2] Before starting with medical management options, a physician should always act in the best interest of the patient by seeking consent.

One of the foundational principles of medical ethics is autonomy.[3] As a result, before beginning any step of medical care, the clinician providing care must respect the patient's decision on treatment 
alternatives by getting informed permission. During this process, the doctor must provide adequate information for the patient to make an informed decision, and so gain proper informed consent. Depending on the procedure's complexity and risk, the information may be modified

Furthermore, the volume of information presented to an individual patient may vary depending on his or her intellectual capacity and level of comprehension. The patient must have the competence to express his or her permission in order to receive legitimate consent for any medical or surgical procedure. As a result, the patient must be competent and autonomous, and consent must be freely given without deception, manipulation, or fraud. [3] During the process of explaining all therapy or inquiry alternatives to the patient, the medical practitioner should be rational, fair, and scientific.

Informed consent should be obtained in writing, ideally with a witness present. When consent cannot be communicated in writing, it must be properly documented with the help of a witness. The majority of patients nowadays are well-informed, wellinformed, and actively participate in medical decision-making. As a result, this procedure offers significant advantages as well as a high degree of patient satisfaction with the overall medical management and outcome. Shared decision making is the ideal paradigm of informed consent, in which both the patient and the clinicians work collaboratively.

The amount of information offered to a patient by a doctor is very low and inconsistent, according to the currently available studies. As a result, the patient is unaware of the benefits and hazards of the treatments/investigations that must be carried out. As a result of this flaw in medical staff, the patient will be able to sue the doctor for any errors, mishaps, and unpreventable and foreseen problems that occur during medical and surgical intervention without sufficient and legal informed permission.

The literature on medical officers' knowledge, attitudes, and practices, as well as informed consent, in Sri Lanka is quite limited. As a result, the study will be timely and necessary in order to fill this gap in Sri Lanka. The purpose of this study was to investigate doctors' knowledge, attitudes, and behaviours about getting informed consent for medical and surgical operations in Kalubowila and Karapitiya Teaching Hospitals.

\section{Methodology}

A cross-sectional descriptive study was conducted among 404 medical officers at Teaching Hospital Karapitiya, and Colombo South Teaching Hospital in Kalubowila, selected on a random basis during a period of six months from 1st May 2018 to November 2018. A self-administered, semistructured questionnaire was formulated to collect data on sociodemographic profile, work experience and knowledge, practices, and attitude of Medical Officers regarding informed consent. Data collection was solely done by the principal investigator after providing informed written consent by all participants. Ethical approval was obtained from the Ethical Review Committee, Teaching Hospital Kalubowila (Ethical approval number 649). The data were analysed by using the latest version of the statistical package for social sciences (SPSS 2020).

\section{Results}

After the data collection, there was a total of 404 medical officers of whom approximately half are from Teaching Hospital Kalubowila $(50.5 \%, \mathrm{n}=$ 204) and the others from Teaching Hospital Karapitiya $(49.5 \%, \mathrm{n}=200)$

\section{A. Socio-demographic data}

Among the study group of 404 medical officers, the majority $57 \% \quad(n=232)$ are males and the sex distribution is shown in Table 01.

Table 01: Gender of the medical officers in the study

\begin{tabular}{lr}
\hline Gender & $\begin{array}{r}\mathbf{N}=\mathbf{4 0 4} \\
\mathbf{n}(\boldsymbol{\%})\end{array}$ \\
\hline Female medical officers & $172(43)$ \\
Male medical officers & $232(57)$ \\
\hline
\end{tabular}

Designation of the majority of the officer's $82 \%$ $(n=332)$ belongs to "Senior House Officers" and the distribution is shown in Table 02 .

Table 02: Designation of medical offices

\begin{tabular}{lr}
\hline Designation & $\begin{array}{r}\mathbf{N}=\mathbf{4 0 4} \\
\mathbf{n}(\boldsymbol{\%})\end{array}$ \\
\hline House officers & $40(10)$ \\
Senior House Officers & $332(82)$ \\
Registrars & $32(08)$ \\
\hline
\end{tabular}

Most of the medical officers in the study group 29\% $(\mathrm{n}=116)$ have had 1- 5 years of working experience followed by $11-15$ years in $24 \% \quad(n=96)$. The working experience of medical officers is shown in Table 03. 
Table 03: Working experience of Medical Officers

\begin{tabular}{lr}
\hline Working experience & $\begin{array}{r}\mathbf{N}=\mathbf{4 0 4} \\
\mathbf{n}(\boldsymbol{\%})\end{array}$ \\
\hline 01year to 05years & $116(29)$ \\
06year to 10years & $94(23)$ \\
11years to 15years & $96(24)$ \\
16years to 20years & $56(14)$ \\
21years to 25years & $26(06)$ \\
26years to 32years & $16(04)$ \\
\hline
\end{tabular}

B. Analysis of the knowledge of the medical officers on informed consent

Knowledge on obtaining informed consent in different procedures was assessed. Among the medical officers in the study, $95 \%(n=384)$ of them knew that informed consent should be obtained in vaginal examination, 95\% $(\mathrm{n}=384)$ in rectal examination, $82 \%(\mathrm{n}=332)$ in blood drawing, $78 \%$ $(\mathrm{n}=314)$ in intravenous treatments, $97 \%(\mathrm{n}=391)$ in surgery and $98 \% \quad(n=394)$ in anesthesia. The responses of the medical officers regarding informed consent in different procedures are shown in Table 04 .

Table 04: the responses that given by medical officers regarding informed consent for vaginal examination, rectal examination, blood drawing, intravenous treatments, surgery and anaesthesia Answer Vaginal Rectal Blood IV SurgeryAnesthe examinaxamina drawinginfusion sia tion tion $\mathrm{N}=404 \mathrm{~N}=404 \quad \mathrm{~N}=404 \quad \mathrm{~N}=404 \quad \mathrm{~N}=404 \quad \mathrm{~N}=404$ n (\%) n (\%) n (\%) n (\%) n (\%) n (\%) \begin{tabular}{lllllll}
\hline No & $04(01)$ & $04(01)$ & $06(01)$ & $06(01)$ & $04(01)$ & $06(01)$
\end{tabular}

Wrong $16(04) 16(04) \quad 48(12) 46(11) \quad 05(01) 03(01)$

Don't $\quad 00(00) 00(00) \quad 18(05) 38(09) \quad 04(01) 01(00)$

Correct $384(95) 398(95) 332(82) 314(78) 391(97) 394(98)$

Among the medical officers in the study group, $71 \%(n=288)$ knew the correct age limit of the patient who can give legally valid consent in Sri Lanka and the different answers given by them is shown in Table 05.

Ninety seven percent $(n=392)$ of the medical officers knew the necessity of the assessment of patient's competency before obtaining informed consent and the answers given by the officers regarding the patient's competency is shown in table 06 .
Table 05: Answers given by officers regarding the age limit for consent

\begin{tabular}{lr}
\hline Age Limit & $\begin{array}{r}\text { N=404 } \\
\text { n (\%) }\end{array}$ \\
\hline Above 14 & $18(05)$ \\
Above 16 & $98(24)$ \\
Above 18 & $288(71)$ \\
\hline
\end{tabular}

Table 06: Answers given by officers regarding patient's competency

\begin{tabular}{lr}
\hline Patients Competency & $\begin{array}{r}\text { N=404 } \\
\text { n (\%) }\end{array}$ \\
\hline no & $12(03)$ \\
Yes & $392(97)$ \\
\hline
\end{tabular}

C. Analysis of the practice and attitude of the medical officers on informed consent

Among the study group, 98\% ( $\mathrm{n}=396)$ of them knew and they apply the knowledge to practice in the case of children and other persons who are incapable of giving consent and 97\% ( $\mathrm{n}=392)$ of them knew that they have to consider the mental state of the patient before obtaining the consent. The answers given by the medical officers regarding the above two conditions are shown in Table 07.

Table 07: Answers given by officers regarding the informed consent what they do in the case of children and others incapable of giving consent and they have to consider the mental state of the Patient before obtaining consent

\begin{tabular}{|c|c|c|}
\hline Answers & $\begin{array}{r}\text { Incapability } \\
\text { of giving } \\
\text { consent } \\
\mathrm{N}=404 \\
\mathrm{n}(\%)\end{array}$ & $\begin{array}{r}\text { Mental state } \\
\text { of the } \\
\text { patient } \\
\mathrm{N}=404 \\
\mathrm{n}(\%)\end{array}$ \\
\hline Wrong & $08(02)$ & $12(03)$ \\
\hline Correct & $396(98)$ & $392(97)$ \\
\hline
\end{tabular}

Among the study group, 96\% ( $\mathrm{n}=348)$ of them knew that they should consider the knowledge of the patients regarding the progress of his /her illness and 93\% $(\mathrm{n}=376)$ of them knew that they must consider the level of understanding of the patients regarding the procedures. The responses of medical officers regarding the above incidents are shown in Table 08. 
Table 08: Answers given by officers, whether they consider the knowledge of the patients regarding the progress of his /her illness and the level of understanding of the patients regarding the procedures.

\begin{tabular}{lrr}
\hline Answers & $\begin{array}{r}\text { Understanding } \\
\text { progress of the } \\
\text { illness }\end{array}$ & $\begin{array}{r}\text { Understandin } \\
\mathbf{g} \text { the } \\
\mathbf{N}=\mathbf{4 0 4}\end{array}$ \\
$\mathbf{n}(\mathbf{\%})$ & $\mathbf{N}=\mathbf{4 0 4}$ \\
& $\mathbf{n}(\boldsymbol{\%})$ \\
\hline No & $56(04)$ & $28(07)$ \\
Yes & $348(96)$ & $376(93)$ \\
\hline
\end{tabular}

Most of the officers $93 \%(n=374)$ were aware that the consent is not necessary for the management in a case of an emergency. Nevertheless, $68 \%(n=276)$ of them were not aware that consent is not required in mandatory immunization process. $85 \%(\mathrm{n}=346)$ of the officers were aware about the necessity of consent in case of routine blood transfusion. Knowledge of the medical officers regarding the consent in emergency procedures is shown in Table 09.

Table 09: Knowledge of the medical officers regarding the necessity of consent in emergency procedures, mandatory vaccination and routine blood transfusion

\begin{tabular}{lrrr}
\hline Answers & $\begin{array}{r}\text { Emergency } \\
\text { Procedure } \\
\mathbf{N = 4 0 4} \\
\mathbf{n}(\boldsymbol{\%})\end{array}$ & $\begin{array}{r}\text { Mandatory } \\
\text { vaccination } \\
\mathbf{N = 4 0 4} \\
\mathbf{n}(\boldsymbol{\%})\end{array}$ & $\begin{array}{r}\text { Blood } \\
\text { transfuse } \\
\mathbf{N = 4 0 4} \\
\mathbf{n}(\boldsymbol{\%})\end{array}$ \\
\hline Wrong & $30(07)$ & $276(68)$ & $346(85)$ \\
Correct & $374(93)$ & $128(32)$ & $58(15)$ \\
\hline
\end{tabular}

The majority of officers in the study $61 \%(\mathrm{n}=246)$ obtain consent in all cases, while $32 \%(\mathrm{n}=130)$ of doctors usually seek consent and $07 \%(n=28)$ of them occasionally take the informed consent before any medical or surgical procedures. $61 \%(n=246)$ of medical offices provides only the most relevant information while providing information for questions of the patients. However, 24\% ( $\mathrm{n}=97)$ of medical officers provide information clearly and briefly and only $15 \%(\mathrm{n}=61)$ provide answers in detail to the patient.

Sometimes, the patient refuses the treatment or investigation options. In case of a similar situation, the majority $61 \%,(n=246)$ of medical offices advise the patient to get a second opinion. However, $24 \%(\mathrm{n}=97)$ of medical officers inform patients briefly and $15 \%(n=61)$ in detail, about possible consequences, if he or she refuses the treatment.
Almost all medical officers $96 \%$, $(n=388)$ believes that the doctor who carries out the treatment or medical procedures is the best person to obtain informed consent from the patient and it is important to know whether the patient understands what the medical officer explained regarding the treatment/procedures.

The Majority 55\% ( $\mathrm{n}=222)$ of medical officers never give a copy of the informed consent to the patient while $10 \%(n=40)$ always give a copy. $14 \%(n=57)$ of them usually give a copy while $21 \%(n=85)$ rarely provide a copy of the informed consent to the patient. The majority of the medical officers $70 \%(n=283)$ only explain common risks while $29 \% \quad(\mathrm{n}=117)$ explain all possible risks and only $01 \%(n=04)$ explain the possible remote risks as well.

\section{Discussion}

Consent is a voluntary agreement given by the patient for any medical procedures. In fact, already in the times of not only the Egyptian civilization, but also the Greek and Roman, documents have been found which show how the doctor's intervention had, in some way, first to be approved by the patient.[2] Therapeutic enlightenment should aid the collaboration of the patient in order to maximize the medical success of the treatment.[2] It is well established by case law and practice that valid consent must meet the following requirements: Competence/capacity of the patient, the consent is given voluntarily, the consent covers the procedure in question and that the patient was informed in making that decision.[4]

According to the results of our study, it is encouraging to note despite not having a system for continuous update of the knowledge, a reasonable number of the doctors have adequate knowledge regarding informed consent.[5] However, some medical officers didn't know the necessity of obtaining informed consent before Blood drawing $(18 \%, \mathrm{n}=73)$, routine blood transfusion $(85 \%, \mathrm{n}=343)$ and IV infusion $(21 \%, \mathrm{n}=85)$. Average $(91 \%, \mathrm{n}=367)$ of them answered correctly to the given questions that inquire their knowledge regarding the informed consent. Hence during the analysis of the knowledge of the medical officers on informed consent, most of them are found to have adequate knowledge.

$29 \%(n=117)$ of medical officers did not know that the age limit of the patient who can give legally valid consent. The figure indicates that a significant percentage of doctors possess insufficient knowledge regarding the concept of consent. Therefore, these populations of medical officers are in danger to become culprits of medical negligence. 
According to most medical officers are well aware that the patient's competency, incapability of giving consent, mental state of the patients, and level of understanding of the patients has to be considered before obtaining consent.

Although most of the medical officers knew that consent was not necessary for emergency management of the patients, they didn't know the consent is not required for mandatory vaccination.[6] This study shows clear evidence regarding awareness of the majority of doctors is limited to some aspects of the consent, and they are not educated or aware in some aspects.

However, our study shows that attitudes and practices of the majority of the medical officers in Karapitiya and Kalubowila Teaching Hospitals are within the accepted levels but in some areas, a considerable number of medical officers have attitudes and practices below the expectations and are very poor in some areas such as giving a copy of informed consent to the patient, that reckons to be one of the basic requirements in this regard.

In a study carried out in Uganda, using a sample of Senior clinicians regarding the knowledge on roles and self-assessment and their compliance to the informed consent process found that a significant proportionate $53 \%$ is at an average level, $40 \%$ at an unsatisfactory level and only $07 \%$ was at the satisfactory level.[7] It was fairly compatible with our study findings.

When we consider the practice and attitudes of doctors, we should accept that there are limitations due to the heavy workload in these hospitals as well. A single medical officer has to be examining many patients during clinics and at wards as well. Therefore, the majority of doctors are working beyond their capacity. During this attempt, the medical officers try to pay attention to the treatment rather than securing the ethical aspects. In fact, in the early 1900s, the Mayo brothers expressed that "The best interest of the patient is the only interest to be considered." Medicine was a dramatically different environment over 100 years ago. It may be more difficult to "live" this message today, with the overlays of regulations, the licensing/credential bodies, codes of conduct, and organizational cultures, but we must in order to sustain our humanity in medicine.[8]

Due to limited time to see the patient, most of the time medical officer takes a decision on behalf of the patient for the best interest of the patient. In fact, in the early 1900s, the Mayo brothers expressed that "The best interest of the patient is the only interest to be considered." Medicine was a dramatically different environment over 100 years ago. It may be more difficult to "live" this message today, with the overlays of regulations, the licensing/credential bodies, codes of conduct, and organizational cultures, but we must sustain our humanity in medicine.[9] Until this treatment or procedure is successful and cures the patient, it will create no issues. But once the expected outcome is not achieved, the medical officer faces many troubles including medical malpractice charges.[10]

Although some specialized programs are targeting selected populations (Eg: Postgraduate trainees), there are no such programs that are carried out regularly to improve the knowledge of the rest of the medical officers regarding the ethical aspect in Sri Lanka. Therefore, it is highly recommended that some sort of continuous professional development programs and knowledge updating programs covering medical ethics need to be introduced at each hospital level.

\section{Conclusions}

According to the results of our study, it is encouraging to note that despite not having a system for continuous update of the knowledge, a reasonable number of the doctors were having decent knowledge regarding the informed consent. During the analysis of the knowledge of the medical officers on informed consent, most of them are found to have adequate knowledge, but in some areas a considerable number of medical officers were having attitudes and practices below the required level, and very poor in some areas such as giving a copy of informed consent to the patient, that known to be one of the basic requirements in this regard.

There are no such programs that are being carried out regularly to improve the knowledge of the practising medical officers regarding the ethical aspects in Sri Lanka. Therefore, it is highly recommended some sort of continuous professional development programs and knowledge updating programs covering medical ethics at each hospital level.

\section{Disclosure statement}

Conflicts of interest: The authors declare that they have no conflicts of interest.

\section{Funding: None}

\section{References}

1. Jukic M, Kvolik S, Kardum G, Kozina S, Tomic Juraga A. Knowledge and practices of obtaining informed consent for medical procedures among 
specialist physicians: questionnaire study in 6 Croatian hospitals. Croatian medical journal. 2009 Dec 15;50(6):567-74.

2. Yousuf RM, Fauzi AR, How SH, Rasool AG, Rehana K. Awareness, knowledge and attitude towards informed consent among doctors in two different cultures in Asia: a cross-sectional comparative study in Malaysia and Kashmir, India. Singapore medical journal. 2007 Jun;48(6):559-65.

3. Perera UCP. Principles of medical law for medical undergraduates and practitioners. Southern province of Sri Lanka: Ministry of health, law and order; 2016.

4. Verma S. Exemption from informed consent: When it is possible in investigational product and drug trials?. Saudi Journal of Anaesthesia. 2021 Oct;15(4):428-30.

5. Azizian J, Dalai C, Adams MA, Murcia A, Tabibian $\mathrm{JH}$. Medical professional liability in gastroenterology: definitions, trends, risk factors, provider behaviors, and implications. Expert Rev Gastroenterol Hepatol. 2021;15(8):909-18. doi:10.1080/17474124.2021.1940957

6. Giubilini A. The Ethics of Vaccination [Internet]. https://link.springer.com/book/10.1007/978-3-03002068-2 Accessed 05 December 2021

7. Kajja I, Bimenya GS, Sibinga CT. Informed consent in blood transfusion: Knowledge and administrative issues in Uganda hospitals. Transfusion and Apheresis Science. 2011 Feb 1;44(1):33-9.

8. Farhat W, Qiam F, Shah SM, Khan M, Khan H. Informed consent in dentistry: Perceived importance and limitations in Khyber Pukhtunkhwa. Journal of Khyber College of Dentistry. 2013 Jun;3(2):14-9.

9. Powell SK. The Best Interest of the Patient Powell SK. The best interest of the patient. Professional Case Management. 2015;20(2):61-2.

10. Bal BS. An introduction to medical malpractice in the United States. Clinical orthopaedics and related research. $2009 \mathrm{Feb}$; 467(2):339-47. 